\section{Multidetector CT des Herzens: Erste Klinische Anwendung einer retrospektiv EKG-gesteuerten Spirale mit optimierter zeitlicher und örtlicher Auflösung zur Darstellung der Herzkranzgefäße}

Zusammenfassung. Zielsetzung: Die deutlich verbesserte zeitliche und örtliche Auflösung der neuen Multidetektortechnologie in der Computertomographie eröffnet auch neue Möglichkeiten der nichtinvasiven Herzbildgebung. Ziel dieser Arbeit ist es, Methoden und erste Ergebnisse der schnellen Mehrschicht Spiral CT für die kardiale Bildgebung mittels EKG-synchronisierter Spiral-Akquisition darzustellen. Material und Methode: Insgesamt 10 Patienten (5 nativ zur Quantifizierung des Koronarkalks, 5 mit Kontrastmittel zur Darstellung der Koronargefäße) wurden an einem schnellen Mehrschicht CT System mit simultaner Aufnahme von 4 Schichten und $0,5 \mathrm{~s}$ Rotationszeit (Siemens Somatom Volume Zoom) untersucht. Kontinuierliche Spiraldatensätze des gesamten Herzvolumens werden zusammen mit dem EKG aufgenommen und mit dedizierten Spiral-Algorithmen bei 250 ms zeitlicher Auflösung rekonstruiert. Dreidimensionale Bilddatensätze konnten in einer frei wählbaren Phase des Herzzyklus (z.B. diastolische Phase) aus überlappend rekonstruierten Einzelschichten zusammengesetzt werden. Durch die Rekonstruktion mehrerer Bilddatensätze in verschiedenen Phasen des Herzzyklus wurde die Herzfunktion analysiert. Ergebnisse: Ein Spiral-Datensatz des gesamten Herzvolumens konnte in einer Atemanhalteperiode aufgenommen werden. Bei $3 \mathrm{~mm}$ Schichtdicke lagen die Scanzeiten zwischen 12-17 s, für hochaufgelöste CT-Angiographien der Koronararterien mit 1,25 mm Schichtdicke zwischen 2535 s. Kalkplaques ließen sich ohne Artefakte darstellen. Ein dreidimensionales Bild des Herzens und der Koronararterien konnte ohne Bewegungsartefakte und mit hoher räumlicher Auflösung in der diastolischen Phase des Herzzyklus erzeugt werden. Schlußfolgerungen: Die schnelle Mehrschicht-SpiralCT mit retrospektiv EKG-synchronisierter Spiralrekonstruktion (EKG-Gating) eignet sich für die dreidimensionale und funktionelle Bildgebung des Herzens, insbesondere für die hochaufgelöste Abbildung kalzifizierter Plaques und für die CTA der Koronararterien.

Schlüsselwörter: Mehrschicht-CT - Spiral-CT - Cardio CT - retrospektives EKG-Gating

Cardiac Multidetector-Row CT: Retrospectively ECG-Gated Spiral with Optimized Temporal and Spatial Resolution: First Clinical Results. Purpose: The significantly improved temporal and spatial resolution of Multidetector-Row CT opens

Fortschr Röntgenstr 2000; 172: 429-435

(c) Georg Thieme Verlag Stuttgart · New York

ISSN 1438-9029
A. F. Kopp ${ }^{1}$, B. Ohnesorge'2, T. Flohr², C. Georg',

S. Schröder ${ }^{3}$, A. Küttner ${ }^{1}$, J. Martensen ${ }^{1}$, C. D. Claussen ${ }^{1}$

${ }^{1}$ Radiologische Universitätsklinik Tübingen,

Abt. für Radiologische Diagnostik, Tübingen

2 Siemens Medizinische Technik, Forchheim

${ }^{3}$ Medizinische Universitätsklinik Tübingen, Abt. III Kardiologie, Tübingen up new possibilities for cardiac imaging. A method with retrospectively ECG-gated spiral acquisition is presented. Materials and Methods: A total of 10 patients underwent cardiac CT on a fast multi-slice CT system with 4 simultaneously acquired slices and $0.5 \mathrm{~s}$ rotation time (Siemens Somatom Volume Zoom). Continuous spiral data of the entire heart volume ( 5 studies precontrast for calcium scoring, 5 studies with contrast) were acquired together with the patient's ECG and reconstructed with dedicated spiral algorithms providing $250 \mathrm{~ms}$ temporal resolution. Three-dimensional image data sets were built up from overlapping slices that were reconstructed in an arbitrary, user-defined phase of the heart cycle (e. g., diastolic phase). To evaluate the capability of the method for functional imaging, complete image volumes were reconstructed from the same spiral data set in different phases of the heart cycle. Results: Within a single breath-hold, a spiral data set of the entire heart volume could be acquired. Typical scan times for standard examinations with 3-mm slice width were 12-17 s, and for high-resolution CT angiographies of the coronary arteries with $1.25-\mathrm{mm}$ slice width about 25-35 s. Motion-free reconstruction of the heart and coronary arteries with high spatial resolution were possible in the diastolic phase of the heart cycle. Multiphase reconstructions from the same spiral scan data set were possible. Conclusions: Fast multi-slice spiral CT with retrospectively ECG-gated spiral reconstruction is well suited for threedimensional and functional imaging of the heart, especially for high-resolution imaging of calcified coronary plaques and CT-angiography of the coronary arteries.

Key words: Multi-slice CT - Spiral-CT - Cardio CT - Retrospective ECG-Gating

\section{Einleitung}

Die koronare Herzkrankheit (KHK) ist die Haupttodesursache in Europa und den USA. Annähernd 600000 Todesfälle in Europa waren 1998 durch eine koronare Herzerkrankung bedingt. Auch wenn viele Patienten über charakteristische Beschwerden berichten, haben doch annähernd die Hälfte der Patienten keine Symptome vor einem plötzlichen Herztod. Daher wurde früh die Forderung nach einem Screening-Test laut, der es erlauben sollte, Patienten mit einem erhöhten Risiko zu erkennen. Seit 1983 steht hierfür das Elektronenstrahl-CT zur Verfügung, mit dem Kalkablagerungen in den Herzkranzgefäßen dargestellt und gemessen werden können. Der Stellenwert dieser Quantifizierung von Kalk in den Koronararterien wird jedoch noch immer von vielen Arbeitsgruppen kontrovers diskutiert, insbe- 
sondere die schlechte Reproduzierbarkeit der errechneten Scores wird kritisiert [1]. Darüber hinaus erlaubt die native Scoring Untersuchung keine direkte Visualisierung von Engstellen in den Koronararterien. Hierfür ist weiterhin eine selektive invasive Koronarangiographie nötig. Moshage et al. stellten 1995 die i.v. kontrastverstärkte nichtinvasive EBCTAngiographie der Koronargefäße vor [2]. Neben technischen Limitierungen (minimale Schichtdicke $3 \mathrm{~mm}$, nur prospektive Triggerung möglich) wird jedoch die breite klinische Anwendung des EBT für die Herzdiagnostik durch die geringe Zahl der installierten Geräte limitiert. Hierfür dürften auch der hohe Wartungsaufwand sowie bauliche und investive Nachteile der EBCT verantwortlich sein. Weltweit sind daher derzeit nur 110 Geräte im Einsatz [3].

Seit wenigen Monaten sind CT-Geräte der sogenannten 3.Generation verfügbar, die mit Multidetektortechnologie und schneller Rotation der Röhre die Leistungsdaten der Elektronenstrahlcomputertomographen erreichen und in vielen Bereichen sogar übertreffen [4].

Im folgenden soll gezeigt werden, wie die Multidetektortechnologie und dafür speziell entwickelte Rekonstruktionsalgorithmen für die Bildgebung des Herzens eingesetzt werden können und welche klinischen Möglichkeiten sich aus der neuen Technologie ergeben.

\section{Material und Methoden}

\section{Patienten}

Im Rahmen einer Pilotstudie wurden 10 Patienten untersucht: 5 ohne Kontrastmittel zur Quantifizierung der Kalkablagerungen in den Herzkranzgefäßen. 5 Patienten wurden im hochauflösenden Modus mit Kontrastmittel untersucht. Einschlußkriterium war der Verdacht auf eine koronare Herzerkrankung bei Patienten älter als 35 Jahre. Ausschlußkriterien waren eine bekannte Niereninsuffizienz (Kreatinin > 1,5 mg/dl), instabile Angina pectoris, bekannte Kontrastmittelallergie, manifeste Hyperthyreose (TSH basal $<0,03 \mathrm{mU} / \mathrm{l}$ ), schwere Leberfunktionsstörungen (GOT, GPT > als der dreifache Normwert), Schwangerschaft und eine Herzinsuffizienz Stadium NYHA III-IV. Jeder Patient gab schriftlich sein Einverständnis. Ein positives Votum der Ethikkommission der Universität lag vor.

\section{Aufnahmeprotokoll}

Alle Patienten wurden auf einem Somatom Volume Zoom ${ }^{\circledR}$ (Siemens, Forchheim) untersucht. Das Gerät verfügt über einen Detektor mit 8 Detektorelementen mit einer Breite von 1, 1,5, 2,5 und $5 \mathrm{~mm}$ von innen nach außen in z-Richtung. Die Rotationsgeschwindigkeit der Röntgenröhre beträgt 0,5 s.

Für die Untersuchung im hochauflösenden Modus mit Kontrastmittel wurde antekubital eine $18 \mathrm{G}$ Kanüle plaziert. Für die Injektion wurde ein Doppelkopfinjektor benützt (Medtron ${ }^{\circledR}$, Saarbrücken), der eine automatische Nachinjektion von physiologischer Kochsalzlösung erlaubt. Zur Bestimmung der Kreislaufzeit wurde ein Testbolus von $20 \mathrm{ml}$ mit $4 \mathrm{ml} / \mathrm{s}$ injiziert, gefolgt von $20 \mathrm{ml}$ physiologischer Kochsalzlösung. $10 \mathrm{~s}$ nach Beginn der Injektion dieses Bolus wurde auf Höhe des Aortenbogens alle $2 \mathrm{~s}$ ohne Tischvorschub eine Aufnahme nach Möglichkeit in Atemstillstand gewonnen bis maximal $120 \mathrm{~s}$ nach Injektion. Die Zeit bis zum maximalen Dichteanstieg in der Aorta ascendens plus einem Korrekturfaktor von 3s wurde als individuelles Delay für die nachfolgende Bolusinjektion verwendet. Diese wurde ebenfalls mit $4 \mathrm{ml} / \mathrm{s}$ durchgeführt. Die applizierte Menge betrug $120-150 \mathrm{ml}$ (370 mg J/ml), gefolgt von $30 \mathrm{ml}$ physiologischer Kochsalzlösung als Nachbolus.

Zwei Modi der retrospektiv EKG-gesteuerten Spiralakquisition (Abb.1) wurden verwendet. Für Standardanwendungen ohne Hochauflösung und native Untersuchungen stand ein Modus mit einer Kollimierung von $4 \times 2,5 \mathrm{~mm}$ und einer effektiven Schichtdicke von $3 \mathrm{~mm}$ zur Verfügung ( $140 \mathrm{kV}, 100 \mathrm{mAs}$ ). Zur Berechnung des Calcium Scores nach Agatston wurden $3 \mathrm{~mm}$ Schichten mit einem Increment von $1,5 \mathrm{~mm}$ berechnet. Der so errechnete Wert wurde durch zwei geteilt. Der hochaufgelöste Modus basierte auf einer Kollimierung von $4 \times 1 \mathrm{~mm}$ bei einer effektiven Schichtdicke von 1,25 mm (140 kV, $300 \mathrm{mAs})$. Dies ermöglicht eine örtliche Auflösung von $9 \mathrm{Lp} / \mathrm{cm}$ in der Bildebene und $6 \mathrm{Lp} / \mathrm{cm}$ in z-Richtung. Der Tischvorschub pro Rotation wurde in beiden Modi an die minimale Herzfrequenz des Patienten angepaßt (Tab. 1, Abb. 2, 3). Der aufgezeichnete Spiraldatensatz enthält durch die kontinuierliche Aufzeichnung alle Herzphasen im Scanintervall. Zur Rekonstruktion eines Bilddatensatzes $\mathrm{zu}$ einem bestimmten Zeitpunkt im Herzzyklus mußten daher die entsprechenden Daten anhand des mitaufgezeichneten EKGs extrahiert werden. Der Zeitpunkt konnte relativ in Prozent eines gesamten RR-Intervalls angegeben werden oder absolut vor (revers) oder nach einer R-Zacke (Abb.1).

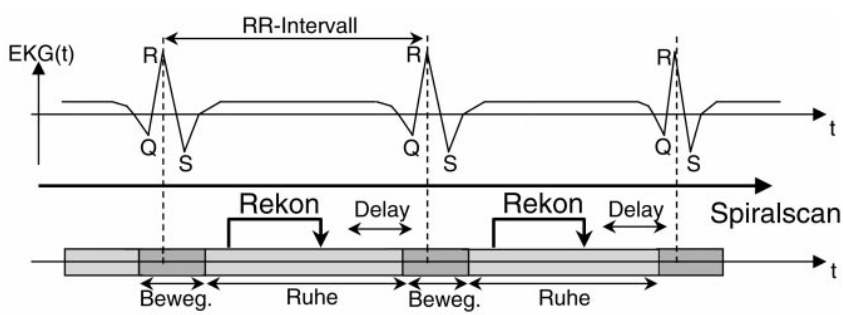

Abb. 1 Prinzip des retrospektiven Gatings: Kontinuierlicher Tischvorschub mit Aufzeichnung des EKG-Signals.

Tab. 1 Adaptation des Pitch an die minimale Herzfrequenz: Pitch < $1500 \mathrm{~ms} / \mathrm{T}_{\mathrm{RR}}$, wobei $\mathrm{T}_{\mathrm{RR}}$ die während des Spiral-Scans maximal zu erwartende Herzzykluszeit darstellt.

\begin{tabular}{lll}
\hline Pulsfrequenz & Herzzykluszeit & Pitch \\
\hline Puls 50 & $\mathrm{~T}_{\mathrm{RR}}=1200 \mathrm{~ms}$ & $<1,25$ \\
Puls 70 & $\mathrm{~T}_{\mathrm{RR}}=860 \mathrm{~ms}$ & $<1,75$ \\
Puls 90 & $\mathrm{~T}_{\mathrm{RR}}=670 \mathrm{~ms}$ & $<2,25$ \\
Puls 110 & $\mathrm{~T}_{\mathrm{RR}}=550 \mathrm{~ms}$ & $<2,75$ \\
Puls 130 & $\mathrm{~T}_{\mathrm{RR}}=460 \mathrm{~ms}$ & $<3,25$ \\
\hline
\end{tabular}

\section{Rekonstruktionsalgorithmen}

Für die Rekonstruktion der Spiraldatensätze mußten spezielle Algorithmen eingesetzt werden, die unter Berücksichtigung der geringen relativen Tischgeschwindigkeit eine optimierte zeitliche Auflösung bieten. Grundlage dieser Rekonstruktionstechnik ist eine Spiralinterpolation für Mehrschichttechnik mit einer Teilscanrekonstruktion. Die 4 Detektorzeilen werden mit 




Abb. 2 Schema der Datenakquisition in Subvolumina: Das Gesamtvolumen entsteht durch Addition von Subvolumina aus aufeinanderfolgenden Herzzyklen.

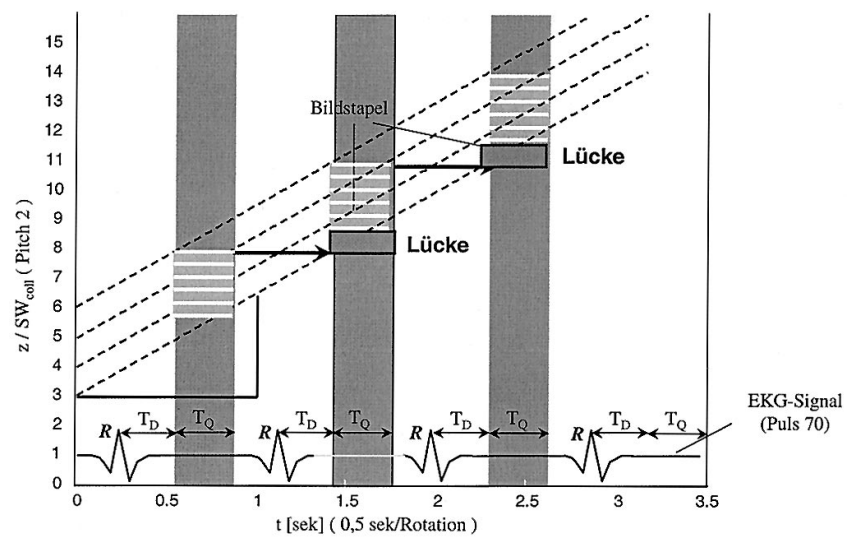

Abb. 3 Tischgeschwindigkeit zu schnell für aktuelle Herzfrequenz. Lücken in der z-Achsenabdeckung entstehen.

zunehmender Zeit t und zunehmendem Projektionswinkel $\alpha$ entlang der z-Achse des Patienten verschoben. Die Mehrschichtspiral-Gewichtung liefert für jede Bildposition einen planaren Einzelschicht-Teilumlaufdatensatz aus einem Partialscan von $240^{\circ}$ ( $2 / 3$ einer Rotation) der 4 Detektorzeilen um eine gegebene z-Position. Für jeden Projektionswinkel $\alpha$ innerhalb des Mehrschichtspiraldatensatzes wird eine lineare Interpolation zwischen zwei Detektorreihen durchgeführt, die dieser selektierten z-Position am nächsten sind [4]. Die Wichtung der jeweiligen Interpolation wird bestimmt durch den Abstand der jeweiligen Subprojektion zur gegebenen z-Position. Dieser für eine bestimmte z-Position so berechnete planare TeilscanDatensatz wird mit einem optimierten Einzeilen-TeilumlaufAlgorithmus rekonstruiert. Hiermit läßt sich im Zentrum des Meßfelds eine zeitliche Auflösung in Größenordnung der Hälfte der Rotationszeit erreichen.

Für das beschriebene Spiral-Rekonstruktionsverfahren gilt eine feste Beziehung zwischen der Halbwertsbreite SW des Schichtempfindlichkeitsprofils (definiert als Schichtdicke) und der kollimierten Schichtdicke $\mathrm{SW}_{\text {koll }}$ einer Einzelschicht des Mehrschichtdetektors, unabhängig von derTischvorschubgeschwindigkeit.

$\mathrm{SW}=1.25 \mathrm{SW}_{\mathrm{koll}}$
Zur Bestimmung des optimalen Rekonstruktionszeitpunktes wurden Bilddatensätze im zeitlichen Abstand von $50 \mathrm{~ms}$ berechnet. Für optimierte 2D- und 3D-Darstellungen wurden alle Rekonstruktionen mit einer Überlappung von $50 \%$ berechnet (Rekonindex 0,6 $\mathrm{mm}$ für Schichtdicke $1,25 \mathrm{~mm}$ und $1,5 \mathrm{~mm}$ für Schichtdicke $3 \mathrm{~mm}$ ). Aus den axialen Einzelschichten wurden multiplanare Reformatierungen, maximale Intensitätsprojektionen, Shaded-Surface-Display und Volume-Rendering-Darstellungen auf einer 3D Virtuoso ${ }^{\circledR}$ (SIEMENS, Forchheim) generiert. Bei drei Patienten wurde eine virtuelle Angioskopie der proximalen Segmente der rechten und linken Kranzarterie durchgeführt. Die so generierten Visualisierungen wurden von zwei unabhängigen Auswertern analysiert. Diese mußten in einer dreistufigen Skala die Bildqualität und evtl. Bewegungsartefakte der proximalen, medialen und distalen Abschnitte der Koronargefäße beurteilen.

\section{Ergebnisse}

In der Gruppe der mit dem nativen Standardprotokoll untersuchten Patienten lag die mittlere Herzfrequenz bei $72 /$ min (minimal 53/min, maximal 82/min). Die mittlere Scandauer betrug damit $14 \mathrm{~s}$ (minimal $12 \mathrm{~s}$, maximal $17 \mathrm{~s}$ ). Über diese Zeitspanne konnten alle Patienten die Luft ohne vorheriges Training problemlos anhalten. Bei allen Patienten $(n=5)$, die untersucht wurden, konnten Kalkablagerungen in den Herzkranzgefäßen nachgewiesen werden. Der mittlere Ca Score nach Agatston betrug 300 [5]. Drei von 5 Patienten wiesen einen Score oberhalb der 75\% Perzentile auf [6]. Die rekonstruierten Bilder mit einer effektiven Schichtdicke von $3 \mathrm{~mm}$ waren frei von sichtbaren Bewegungsartefakten. Die Kalkablagerungen in den Herzkranzgefäßen zeigten keine Streifen- oder Sternartefakte (Abb.4). Die überlappende Schichtfolge führt zu einer deutlichen Reduktion von Partialvolumeneffekten und erlaubt eine Beurteilung der Struktur der Plaques in z-Richtung.

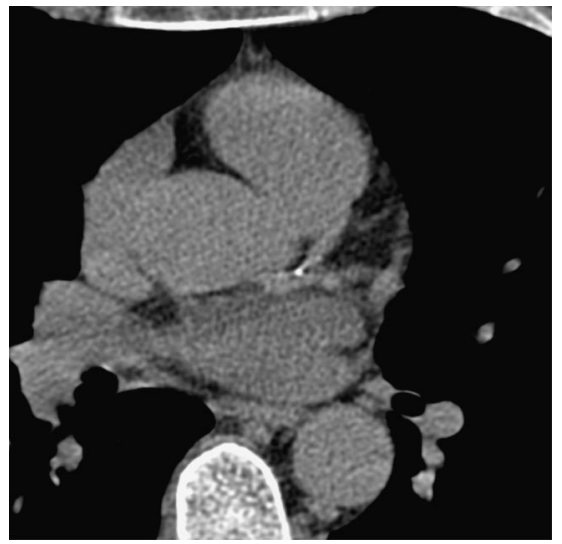

Abb. 4 Axiale Schicht auf Höhe des Ramus interventricularis anterior der linken Koronararterie mit kleinem Kalkplaque im linken Hauptstamm. Schichtdicke $3 \mathrm{~mm}$, Inkrement $1 \mathrm{~mm}$, Pitch 1,5, Rotationszeit $0,5 \mathrm{~s}$, Rekonstruktion mit $250 \mathrm{~ms}$ zeitlicher Auflösung in der Diastole bei $\mathrm{T}_{\mathrm{rev}}=500 \mathrm{~ms}$.

In der Gruppe der Patienten, die mit dem hochauflösenden CTAModus untersucht wurden, lag die mittlere Herzfrequenz vor der Untersuchung bei 60/min (minimal 45/min, maximal 75/ min). Unter Kontrastmittelinjektion stieg die durchschnittliche Herzfrequenz um 20 Schläge/min auf durchschnittlich $80 / \mathrm{min}$ an. Die Tischgeschwindigkeit wurde an die minimale Herzfrequenz angepaßt. Die mittlere Scandauer zur Darstellung des Herzens von den Koronarostien bis zum Apex betrug $27 \mathrm{~s}$ (minimal $25 \mathrm{~s}$, maximal $35 \mathrm{~s}$ ). Lediglich ein Patient konnte den Atem nicht über die gesamte Scandauer anhalten. Bei allen 5 


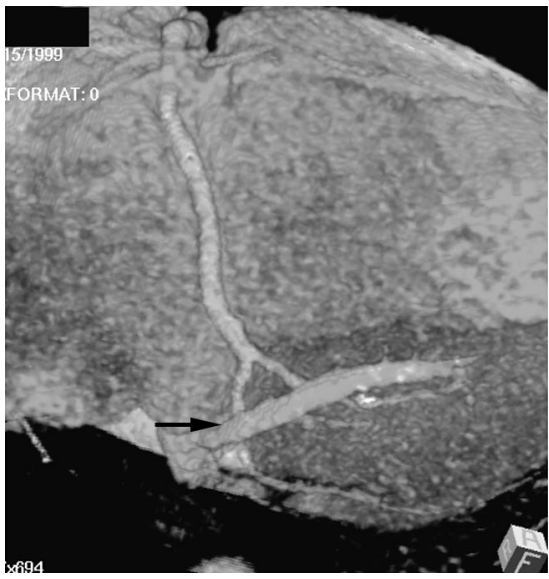

Abb. 5 Volume Rendering Darstellung der rechten Koronararterie (Ansicht von kaudal). Darstellung des Lumens bis Segment 3. Auch eine Vene findet sich im Blickfeld (Pfeil). Aufnahmeparameter: Schichtdicke 1,25 mm, Inkrement $0,5 \mathrm{~mm}$, Pitch 1,5, Rotationszeit $0.5 \mathrm{~s}$, Rekonstruktion mit 250 ms zeitlicher Auflösung in der Diastole bei $\mathrm{T}_{\text {rev }}=$ $600 \mathrm{~ms}$. a

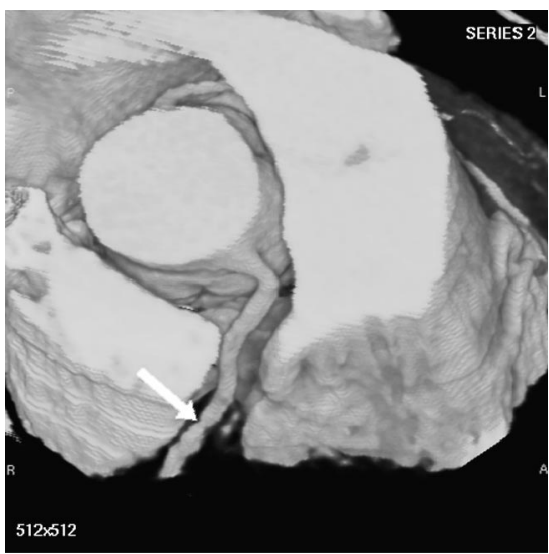

b

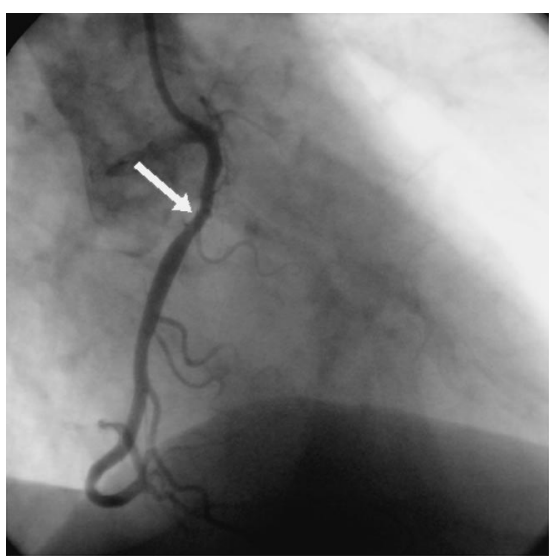

Abb. 6 (a, b) 60jähriger Patient. Z.n. PTCA Segment 1 der rechten Koronararterie. Darstellung des proximalen Abschnitts der rechten Koronararterie. Vergleich mit konventioneller Koronarangiographie. Darstellung der Offenheit des Gefäßes mit nicht relevanter Reststenose (ca. $20 \%$ ) in der MDCTA mit guter Korrelation zum Herzkatheter (Pfeile). Aufnahmedicke $1,25 \mathrm{~mm}$, Inkrement 0,5 mm, Pitch 1,5, Rotationszeit $0,5 \mathrm{~s}$, Rekonstruktion mit $250 \mathrm{~ms}$ zeitlicher Auflösung in der Diastole bei $\mathrm{T}_{\text {rev }}=450 \mathrm{~ms}$. parameter: Schicht-

Patienten gelang die Darstellung der linken sowie der rechten Herzkranzarterie bis mindestens zum Übergang vom mittleren zum peripheren Drittel des jeweiligen Gefäßes (Abb. 5 - 7). Der optimale Rekonstruktionszeitpunkt war für die einzelnen Gefäße unterschiedlich und hing von der jeweiligen Herzfrequenz ab (Abb.8). Für die rechte Koronararterie war ein Rekonstruktionszeitpunkt in der frühen Diastole günstiger (Abb. 5,9). Die dünne Schichtführung mit der optimalen Überlappung erlaubte auch die Generierung von virtuellen Endoskopien der Herzkranzgefäße (virtuelle Koronarangioskopie) (Abb. 7).

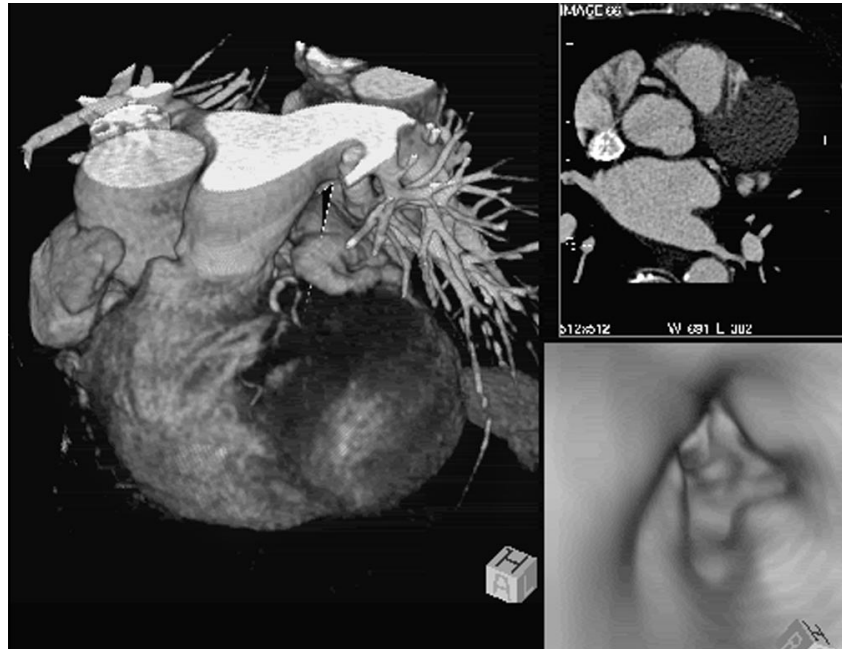

Abb. 7 Virtuelle Angioskopie des Ramus circumflexus der linken Koronararterie. Die Auswahl von geeigneten Transferfunktionen erlaubt die direkte Visualisierung des Lumens. Die Volume-Rendering-Übersichtsdarstellung sowie das axiale Schichtbild zeigen die Position des virtuellen Endoskops.

Diese wurden auf einer Postprocessing-Workstation erstellt (3D Virtuoso - , VA30, Siemens, Forchheim). Pro Patient wurden hierfür 30-45 Minuten benötigt.

Durch Rekonstruktion zu den entsprechenden Zeitpunkten konnten Bilder in der Endsystole sowie der Enddiastole generiert werden. Dies erlaubte die Berechnung von Funktionsparametern wie z.B. der Ejektionsfraktion, Myokardwandverdickung oder Kontraktilität (Abb.10).

\section{Diskussion}

Die hohe Prävalenz der koronaren Herzerkrankung und ihre große volkswirtschaftliche Bedeutung ließen mit der Entwicklung der Schnittbildverfahren die Hoffnung aufkommen, ein nichtinvasives Verfahren auch für die Herzkranzgefäße zur Verfügung zu haben. Während in anderen Körperregionen nichtinvasive CT- und MR-Angiographien bereits in die klinische Routine Eingang gefunden haben, ist die Bildgebung der Koronargefäße durch den komplexen Verlauf dieser Gefäße, die Kontraktionsbewegungen des Herzens und die Atmung deutlich erschwert. In der MR Tomographie wurden durch neue Gating- und Sequenztechniken bedeutende Erfolge erzielt, breiten Eingang in die Routinediagnostik der koronaren Herzerkrankung hat das Verfahren jedoch noch nicht gefunden [7]. Die Elektronenstrahl-Computertomographie ist seit 1983 verfügbar und wird v.a. in den USA als Screeningmethode zur Detektion und Quantifizierung kalzifizierter Plaques in den Koronararterien eingesetzt. Moshage u. Achenbach führten die EBCT-Angiographie ein. Wichtige limitierende Faktoren des EBT sind jedoch die mangelhafte Reproduzierbarkeit der CaScores, die für Arrhythmien empfindliche prospektive Triggerung sowie die in z-Achse ungenügende räumliche Auflösung [8].

Mit der Entwicklung der Mehrschicht Spiral CT wurde die Datenakquisition für CT-Geräte der dritten CT-Generation mit rotierender Röntgenröhre-Detektoreinheit gesteigert. Dies kann zur Optimierung der räumlichen und zeitlichen Auf- 


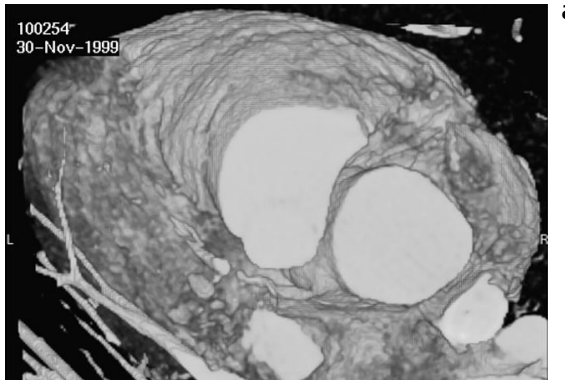

a Abb. 8 Vergleich unterschiedlicher Rekonstruktionszeitpunkte im Herzzyklus für den Ramus interventricularis anterior (RIVA) der linken Kranzarterie: 250 (a), 350 (b), 450 (c) und $550 \mathrm{~ms}$ (d) absolut revers. Die Rekonstruktion bei

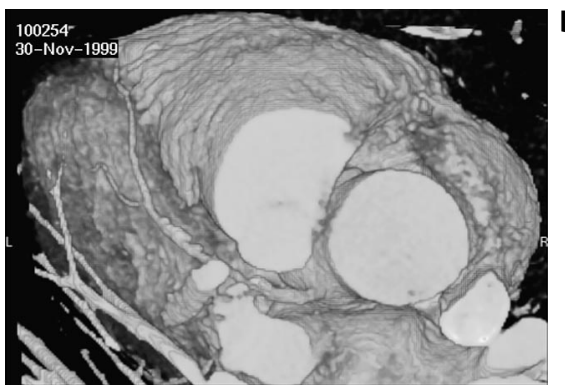

b 350 ms zeigt die Gefäßkonturen des RIVA am besten.

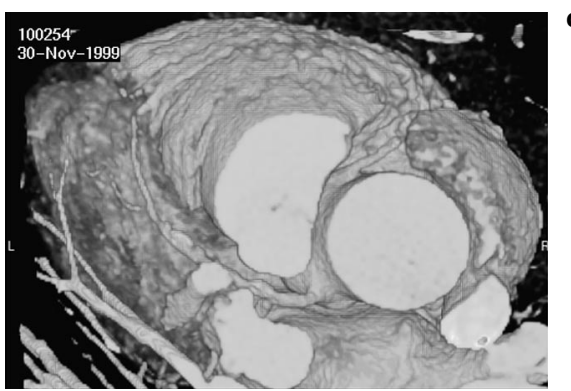

C

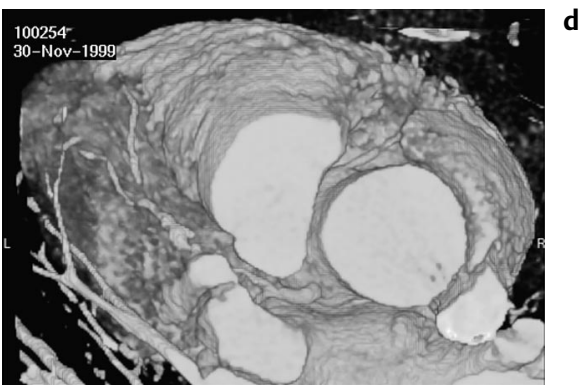

lösung für die Herzbildgebung genutzt werden [9]. Die hier vorgestellte Methode erlaubt die Abbildung des kompletten Herzens mit 1,25 mm dicken Schichten mit einer zeitlichen Auflösung von $250 \mathrm{~ms}$ in einer einzigen Atemanhaltephase.

Für die Anwendung am Herzen auf einem Multidetektorgerät sind konventionelle Rekonstruktionsalgorithmen nicht verwendbar. Standardalgorithmen für die Mehrschicht-Spiralrekonstruktion sind hinsichtlich der bestmöglichen Ausnutzung der applizierten Strahlendosis optimiert [10,11]. Dies bedingt, daß sich die zeitliche Auflösung bei geringem Tischvorschub (Vorschub pro Umdrehung < Gesamtbreite des Detektors) verschlechtert, d.h. die zeitliche Auflösung vom gewählten Pitch abhängig ist [12]. Dies gilt im besonderen für kleine Pitchwerte (<4) mit überlappender Datenaufnahme. Hier wird eine z-Position nacheinander von mehreren Detektorreihen erfaßt. Hat sich in dieser Zeit die z-Position (z. B. durch Atmung) verändert, so kommt es zu einer Fehlregistrierung. Da für die

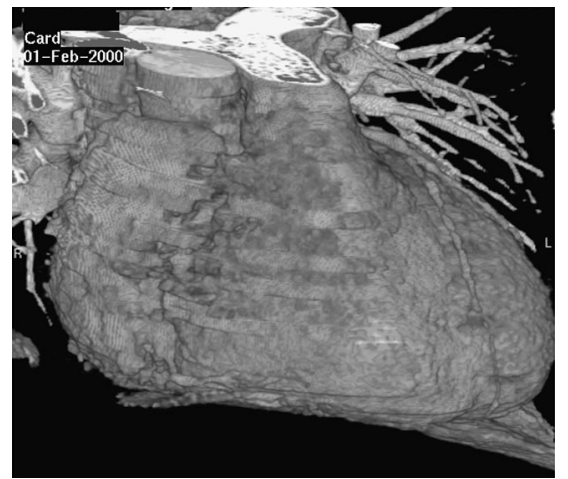

a Abb. 9 Volume Rendering Darstellung der rechten Koronararterie. Links mit einem reversen Gating Delay von $300 \mathrm{~ms}$ (a), rechts von $600 \mathrm{~ms}(\mathbf{b})$. In der Rekonstruktion der späten Diastole (a, $300 \mathrm{~ms}$ ) ist die rechte Kranzarterie kaum abgrenzbar. Eine Rekonstruktion in der frühen Dia-

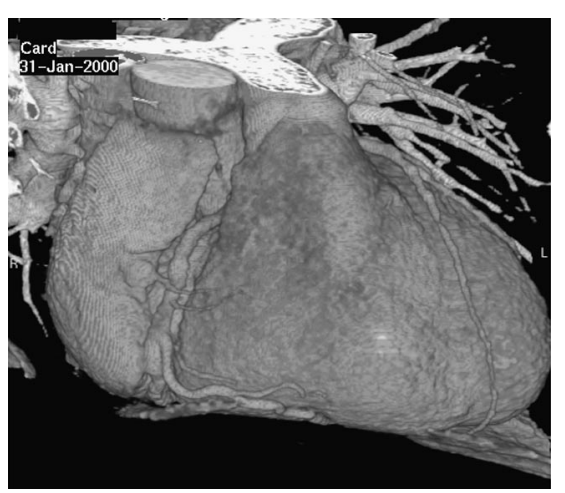
b stole (b, $600 \mathrm{~ms})$ ist deutlich günstiger als in der Enddiastole.

Herzbildgebung eine geringe Tischgeschwindigkeit mit überlappender Datenaufnahme notwendig ist, müssen die spezifischen Cardio-Rekonstruktionsalgorithmen diesen Umstand berücksichtigen und tragen dem geringen relativen Tischvorschub Rechnung.

Konventionelle Teilscan-Rekonstruktionstechniken erreichen eine zeitliche Auflösung in der Größenordnung der Zeitdauer eines Teilumlaufes, d.h. ca. 2/3 der Rotationszeit entsprechend $340 \mathrm{~ms}$ bei einer Rotationszeit von $500 \mathrm{~ms}$ [13]. Die neuen von uns verwendeten Algorithmen erreichen die Größenordnung von ca. 1/2 der Rotationszeit, d.h. bei einer Umlaufzeit von $500 \mathrm{~ms}$ ca. $250 \mathrm{~ms}$. Dies wird möglich durch eine Transformation der Daten von Fächerstrahlgeometrie in Parallelstrahlgeometrie durch ein sogenanntes Rebinningverfahren [14,15]. Die vollständigen Parallelstrahl-Projektionen eines Projektions-Winkelbereichs von $180^{\circ}$ sind zur Bildrekonstruktion ausreichend.

Zur Datenaufnahme wurde bei beiden Modi die Technik des retrospektiven Gatings eingesetzt. Die Aufnahme der Bilddaten geschieht in Spiraltechnik bei kontinuierlichem Tischvorschub und gleichzeitiger Aufzeichnung des EKG-Signals des Patienten. In jedem Herzzyklus wird ein kleines Bild-Volumen akquiriert, das aus überlappenden Einzelschichten mit festem Inkrement besteht. Das Gesamtvolumen entsteht somit durch Addition dieser Subvolumina aus aufeinanderfolgenden Herzzyklen. Eine kontinuierliche Volumenabdeckung ist aber nur dann gewährleistet, wenn der Tischvorschub so an die Herzrate angepaßt wird, daß alle Sub-Volumina in der z-Richtung überlappen und keine Lücken entstehen (Tab.1). Diese Bedingung wird durch eine Begrenzung des Pitches sichergestellt:

Pitch $<1500 \mathrm{~ms} / \mathrm{T}_{\mathrm{RR}}$ 
a

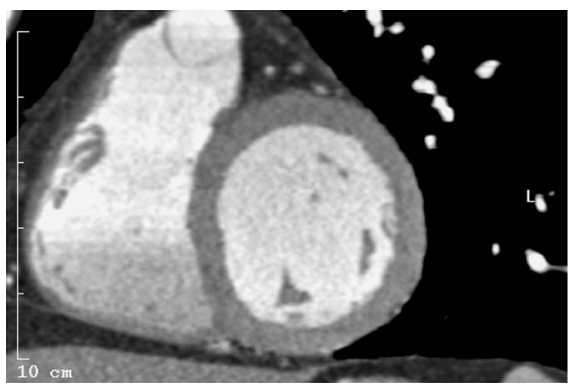

b

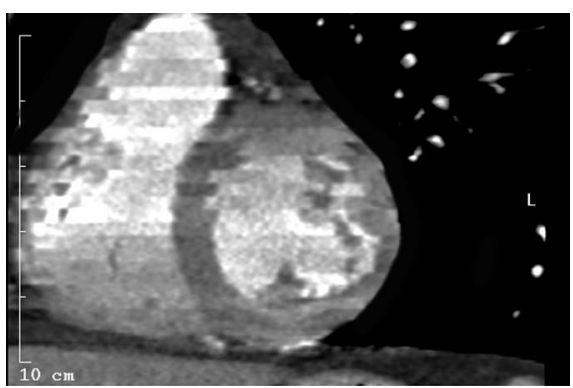

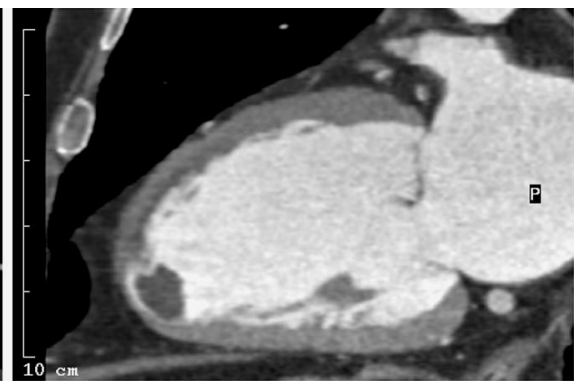



Abb.10 MPRs in der kurzen und langen Herzachse der Rekonstruktionen in der Enddiastole (a) und in der Endsystole (b) erlauben die Berechnung von Funktionsparametern wie endsystolisches und enddiastolisches Volumen, Ejektionsfraktion und Auswurfvolumen. Im Längsschnitt erkennt man in der Diastole die geöffnete Mitralklappe, in der Systole die geschlossene Klappe. In der langen Herzachse ist ein subendokardialer Infarkt in der Vorderwand sowie im Apex ein kleines Aneurysma mit einem Thrombus zu erkennen.
Der Pitch ist dabei definiert als der Tischvorschub pro Umdrehung, bezogen auf die kollimierte Schichtdicke $S_{\text {koll }}$ einer Einzelschicht des Mehrschichtdetektors, $\mathrm{T}_{\mathrm{RR}}$ ist die während des Spiral-Scans maximal zu erwartende Herzzykluszeit. Für übliche Herzraten von $60-100 \mathrm{~min}^{-1}$ ergeben sich so Pitch-Werte von 1,5 bis 2,5. Die Adaptation der Tischgeschwindigkeit an die Herzfrequenz ist für das Rekonstruktionsverfahren grundlegend. Voraussetzung dafür jedoch ist, daß das Schichtempfindlichkeitsprofil als einer der Hauptfaktoren für die Bildqualität vom Pitch weitgehend konstant ist. Dies stellt eine wichtige Neuerung gegenüber Einschicht-Computertomographen dar, die bei höheren Pitchwerten eine zunehmende Verbreiterung des Schichtempfindlichkeitsprofils aufweisen. Ohnesorge et al. konnten zeigen, daß die für die Standardalgorithmen des Multidetektor-CT eingesetzten Algorithmen ein vom Pitch unabhängiges Schichtempfindlichkeitsprofil aufweisen [4]. Dies gilt auch für die hier dargestellten CardioRekonstruktionsalgorithmen. Die effektive Schichtdicke ausgedrückt als FWHM (full width at half maximum) des Schichtempfindlichkeitsprofils beträgt unabhängig vom Pitch das 1,25fache einer Detektorreihe.

Unter Berücksichtigung der hier diskutierten neuen Techniken zeigen unsere vorläufigen Ergebnisse, daß mit einer 4-Schichttechnologie und einer Rotationszeit von 500 ms das menschliche Herz weitgehend bewegungsartefaktfrei in einer Atemanhaltephase dargestellt werden kann. Kalkplaques sind ohne Artefakte darstellbar [16], und die überlappende Rekonstruktion erlaubt eine deutliche Reduktion von Partialvolumeneffekten [17]. Dies verbessert die Reproduzierbarkeit des errechneten Kalkscore signifikant [18]. Bei einer Kollimierung von $1,25 \mathrm{~mm}$ und einer überlappenden Rekonstruktion von $50 \%$ sind CT-Angiographien der Herzkranzgefäße möglich. Die Technik des retrospektiven Gatings erlaubt hierbei für ein gegebenes Gefäß den optimalen Rekonstruktionszeitpunkt zu wählen. Insbesondere scheint für die rechte Herzkranzarterie ein frühdiastolischer Rekonstruktionszeitpunkt günstiger zu sein. Dies läßt sich mit der durch eine Vorhofkontraktion bedingten großen protosystolischen Bewegungsamplitude der rechten Koronararterie erklären [19]. Wird wie in der Elektronenstrahltomographie mit prospektiver Triggerung untersucht, kann ein nicht-optimal gewählter Zeitpunkt im Herzzyklus nicht korrigiert werden. Dies kann Ursache für ausgeprägte Bewegungsartefakte sein und führt dazu, daß einzelne Segmente nicht beurteilt werden können [20].

Die besondere Bedeutung dieser Methodik liegt auch darin, daß Multidetektor-CT-Geräte in kurzer Zeit flächendeckend verfügbar sein werden. Bereits jetzt sind weltweit deutlich mehr Multidetektorgeräte als EBT-Maschinen installiert. Dies liegt in der universellen Einsetzbarkeit dieser Spiralcomputertomographen begründet, d.h. die Durchführung eines Cardio-CTs ist damit nicht mehr auf einige wenige große Zentren mit dedizierten Scannern beschränkt. Das klinische Potential dieser neuen Methode muß noch in größeren Kollektiven evaluiert werden [21]. Es gilt zu zeigen, wie die durch die Volumenerfassung hohe Reproduzierbarkeit und Zuverlässigkeit des Multidetektor-CT-Ca-Scoring klinisch z. B. für Verlaufsuntersuchungen unter medikamentöser Therapie genutzt werden können. Welche klinischen Indikationen sich für die nichtinvasive Multidetektor-CT-Koronarangiographie ergeben, muß die Korrelation mit der konventionellen Koronarangiographie zeigen.

\section{Literatur}

${ }^{1}$ Sechtem U. Electron beam computed tomography: on its way into mainstream cardiology? Eur Heart J 2000; 21: 87 -91

2 Moshage WE, Achenbach S, Seese B. Coronary artery stenoses: three-dimensional imaging with electrocardiographically triggered, contrast agent-enhanced, electron-beam CT. Radiology 1995; 196: $707-714$

${ }^{3}$ Kopp AF. Cardiac Applications of Multidetector-Row CT. San Francisco, California. 1st International Symposium on Multidetector-Row CT. 1999: June 27-28

${ }^{4}$ Ohnesorge B, Flohr T, Schaller S, Klingenbeck-Regn K, Becker C, Schöpf UJ, et al. Technische Grundlagen und Anwendungen der Mehrschicht-CT. Radiologe 1999; 39: 923 -931 
${ }^{5}$ American Heart Association Committee Report. A reporting system on patients evaluated for coronary artery disease. Circulation 1999; 51: 7-34

${ }^{6}$ Rumberger JA, Brundage BH, Rader DJ, Kondos G. Electron beam computed tomographic coronary calcium scanning: a review and guidelines for use in asympatomatic persons. Maye Clin Proc 1999; 74: $243-252$

${ }^{7}$ Wielopolski PA, van Geuns RJM, de Feyter PJ, Oudkerk M. Coronary arteries. Eur Radiol 2000; 10: 12 - 35

${ }^{8}$ Budoff MJ, Georgiou D, Brody A. Ultrafast computed tomography as a diagnostic modality in the detection of coronary artery disease: a multicenter study. Circulation 1996; 93: 898-904

${ }^{9}$ Bruder H, Schaller S, Ohnesorge B, Mertelmeier T. High temporal resolution volume heart imaging with multirow computed tomography. SPIE 1999; 3661: 420-432

${ }^{10} \mathrm{Hu} \mathrm{H}$. Multi-slice helical CT: Scan and reconstruction. Med Phys 1999; 26: 5-18

${ }^{11}$ Flohr T, Schaller S, Ohnesorge B, Klingenbeck-Regn K, Kopp AF. Evaluation of Image Artifacts in Multislice CT. Radiology 1999; 213: 317

${ }^{12}$ Klingenbeck-Regn K, Schaller S, Flohr T, Ohnesorge B, Kopp AF, Baum U. Subsecond multislice computed tomography: basics and applications. Eur J Radiol 1999; 31: 110-124

${ }^{13}$ Parker DL. Optimal short scan convolution reconstruction for fanbeam CT. Med Phys 1982; 9: 254-257

${ }^{14}$ Noo F, Defrise M, Clackdoyle R. Single-slice rebinning method for helical cone-beam CT. Phys Med Biol 1999; 44: 561 - 570

${ }^{15}$ Besson G. New classes of helical weighting algorithms with applications to fast CT reconstruction. Med Phys 1998; 25: 1521 1532

${ }^{16}$ Becker CR, Knez A, Ohnesorge B, Flohr T, Schoepf UJ, Reiser M. Detection and quantification of coronary artery calcifications with prospectively ECG triggered multirow conventional CT and electron beam computed tomography: comparison of different methods for quantification of coronary artery calcifications. Radiology 1999; 213: 351

17 Wexler L, Brundage B, Crouse J, Detrano R, Fuster V, Maddahi J, et al. Coronary artery calcification: pathophysiology, epidemiology, imaging methods, and clinical implications. A statement for health professionals from the American Heart Association. Writing Group. Circulation 1996; 94: 1175-1192

18 Ohnesorge B, Flohr T, Becker CR, Kopp AF, Knez A. Comparison of EBCT and ECG-gated multislice spiral CT: a study of 3D Ca-scoring with phantom and patient data. Radiology 1999; 213: 402

${ }^{19}$ Schermund A, Rensing BJ, Sheedy PF, Bell MR, Rumberger JA. Intravenous electron-beam computed tomographic coronary angiography for segmental analysis of coronary artery stenoses. J Am Coll Cardiol 1998; 31: 1547-1554

${ }^{20}$ Achenbach S, Moshage W, Ropers D, Nossen J, Daniel WG. Value of electron-beam computed tomography for the noninvasive detection of high-grade coronary-artery stenoses and occlusions. N Engl J Med 1998; 339: 1964-1971

${ }^{21}$ Kopp AF, Ohnesorge B, Flohr T, Schroeder S, Claussen CD. Multidetector-row CT for the noninvasive detection of high-grade coronary artery stenoses and occlusions: first results. Radiology 1999; 213: 435

\section{Dr. Andreas F. Kopp}

Radiologische Universitätsklinik Tübingen

Abt. Radiologische Diagnostik

Hoppe-Seyler-Straße 3

72076 Tübingen

Tel. 07071-29-82087

Fax 07071-29-5845

E-mail: andreas.kopp@uni-tuebingen.de 